\title{
Effect of thermal annealing and strain engineering on the fine structure of quantum dot excitons
}

\author{
A. I. Tartakovskii, ${ }^{1}$ M. N. Makhonin, ${ }^{1,2}$ I. R. Sellers, ${ }^{1}$ J. Cahill, ${ }^{1}$ A. D. Andreev, ${ }^{3}$ D. M. Whittaker, ${ }^{1}$ J-P. R. Wells, ${ }^{1}$ \\ A. M. Fox, ${ }^{1}$ D. J. Mowbray, ${ }^{1}$ M. S. Skolnick, ${ }^{1}$ K. M. Groom, ${ }^{4}$ M. J. Steer, ${ }^{4}$ H. Y. Liu, ${ }^{4}$ and M. Hopkinson ${ }^{4}$ \\ ${ }^{1}$ Department of Physics and Astronomy, University of Sheffield, S3 7RH, United Kingdom \\ ${ }^{2}$ Institute of Solid State Physics, Chernogolovka, 142432, Russia \\ ${ }^{3}$ Advanced Technology Institute, University of Surrey, Guildford, Surrey, United Kingdom \\ ${ }^{4}$ Department of Electronic and Electrical Engineering, University of Sheffield, Sheffield S1 3JD, United Kingdom
}

(Received 9 June 2004; published 5 November 2004)

\begin{abstract}
The fine structure splitting of bright exciton states is measured for a range of thermally annealed InGaAs quantum dot (QD) samples with differing degrees of In/Ga intermixing and also for a dot-in-a-well (DWELL) structure. Magnitudes of the fine structure splitting are determined in polarization-resolved differential transmission experiments from measurements of the period of quantum beats observed in QD exciton dynamics. The splitting is found to decrease in structures with weaker strain: both for In/Ga intermixed QD's and also in dots surrounded by strain-reducing layers (DWELL's). Our findings pave the way to the achievement of entangled two photon sources based on emission from individual QD's, currently prevented since the fine structure splitting is larger than the radiative linewidth.
\end{abstract}

DOI: 10.1103/PhysRevB.70.193303 PACS number(s): 78.67.De, 73.21.La, 78.47.+p, 78.55.Cr

In many QD systems the breakdown of a pure-spin picture for exciton states is observed and linearly polarized exciton eigenmodes form split by the electron-hole exchange interaction $^{1-10}$ (see Fig. 1). This prevents the realization of an entangled two photon source based on individual QD's. ${ }^{11,12}$ A splitting smaller than the radiative broadening [of order of a few $\mu \mathrm{eV}$ (Ref. 13)] is required for photon entanglement ${ }^{11}$ in such sources of potential importance for implementation in quantum information systems. ${ }^{14}$

The fine structure splitting arises due to the long range electron-hole exchange interaction, and occurs in systems with lowered in-plane symmetry. ${ }^{4,8,15}$ Due to the reduced atomistic symmetry of zinc blende semiconductors $\left(C_{2 v}\right.$ symmetry), fine structure splitting is expected for self-assembled dots with cylindrical symmetry about the growth axis, although in this case splittings of only up to $\approx 9 \mu \mathrm{eV}$ are predicted, ${ }^{8}$ considerably smaller than those found in most as-grown dot samples (see below). The splitting will then be enhanced by anisotropy in dot shape occurring during crystal growth. In a similar way it is known that the strain field is anisotropic for self-assembled dots, distinguishing the [110] and $[-110]$ directions, even for dots of spherical shape. ${ }^{16,17}$ The strain leads to piezoelectric fields in the samples, and when included in $\mathbf{k} \cdot \mathbf{p}$ theory treatments of electronic band structure, leads to markedly enhanced $p$-state splittings; ${ }^{17}$ the $s$-like ground state is expected to show similarly enhanced splitting when strain and piezoelectric fields are enhanced. In accord with the above arguments, in unstrained dots in a glass matrix, the fine structure is zero. ${ }^{18}$ Further support to the above reasoning is given by recent experiments where externally applied uniaxial strain was shown to produce a large splitting of bright excitons in quantum wells. ${ }^{19}$

Here we present systematic studies of the fine structure splitting in ensembles of InGaAs dots, providing experimental evidence for the factors determining the magnitude of the electron-hole exchange interaction. In particular, we show that $E_{\mathrm{FS}}$ can be accurately tuned in InGaAs dots by post- growth thermal annealing (enhancing the In/Ga intermixing and reducing strain $^{20}$ ) and also reduced considerably in DWELL laser structures where the In-rich dots dots are surrounded by two strain-reducing InGaAs layers. ${ }^{21,22}$ The notable In/Ga intermixing occurring during thermal annealing is shown to result in a smaller $E_{\mathrm{FS}}$. These observations suggest that strain present in dots due to the lattice mismatch of $\mathrm{In}(\mathrm{Ga}) \mathrm{As}$ and GaAs plays an important role in controlling the fine structure splitting. On the other hand, by comparing different QD's grown on GaAs and DWELL structures we find that the depth of the exciton confinement is a less significant factor in determining $E_{\mathrm{FS}}$.

We deduce $E_{\mathrm{FS}}$ from low $T$ measurements of the period of quantum beats in polarization-resolved pump-probe experiments on dot ensembles. In the experiments a circularly polarized pump pulse excites the two linearly polarized exciton modes (split by the electron-hole exchange interaction) with the initial relative phase $\Delta \Theta_{0}=\pi / 2$. Due to the energy split-

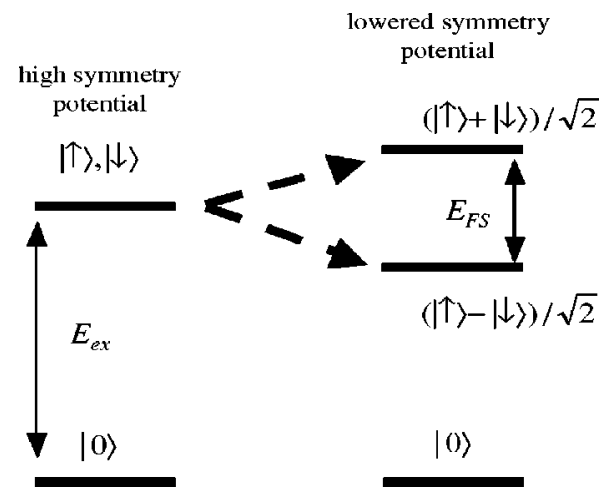

FIG. 1. The diagram of the quantum dot exciton states in the case of the confining potential of a high (left) and low (right) symmetry. $|0\rangle,|\uparrow\rangle$, and $|\downarrow\rangle$ denotes the initial photon and pure spin-up and down exciton states, respectively. Fine structure splitting of the linearly polarized eigenstates in the low symmetry case is denoted $E_{\mathrm{FS}} . E_{\mathrm{ex}}$ denotes exciton energy. 
ting between the exciton modes, the relative phase $\Theta$ evolves after the excitation and exhibits oscillations with period $h / E_{\mathrm{FS}}$. This in turn leads to a periodic variation of the coherent polarization in the sample, which is then probed by a second circularly polarized probe pulse. ${ }^{9,10}$ The oscillations of the coherent polarization in a large ensemble of dots are damped due to the slight dot-to-dot variation of $E_{\mathrm{FS}}$ (which we describe in the model below by a Gaussian of width $\delta$ ). In addition, the coherence of the exciton states $T_{\text {coh }}$ can be further reduced due to scattering processes with, e.g., phonons [insignificant at $T<40 \mathrm{~K}$ (Ref. 10)].

By varying the pump-probe delay the time-dependent differential transmission signal $\Delta P / P$ can be measured ( $P$ laser power transmitted through the sample). $\Delta P / P$ has contributions from (i) the coherent polarization dynamics (responsible for quantum beats) and (ii) incoherent carrier population decay (leading to a slow exponential decay). We find that even in nominally undoped samples (ii) contains separate contributions from neutral and charged dots, ${ }^{10}$ which we introduce into the model via time constants $\tau_{X}$ and $\tau_{\mathrm{ch} X}$, respectively. On the other hand, a charged exciton (in its ground state) does not contribute to (i) due to the suppression of the electron-hole exchange interaction. ${ }^{4,6,7} \mathrm{We}$ can now write $^{23}$

$$
I_{\sigma^{+} \sigma^{ \pm}}=e^{-t / \tau_{X}} \pm e^{-t / T_{\mathrm{coh}}} e^{-\delta^{2} t^{2}} \cos (\omega t)+(1 \pm 1) \frac{A_{\mathrm{ch}}}{4} e^{-t / \tau_{\mathrm{ch} X}},
$$

where $I_{\sigma^{+} \sigma^{ \pm}}$describes the $\Delta P / P$ signal for pumping with a $\sigma^{+}$-polarized pulse and probing with a $\sigma^{ \pm}$-polarized probe. $\omega=E_{\mathrm{FS}} / \hbar$ and $A_{\mathrm{ch}}$ is the weight of the charged dots in the dot ensemble. ${ }^{26}$ In what follows, we will focus on the deduction of $\omega$ from our experimental data, which we show can be achieved with accuracy better than $\pm 10 \%$.

We studied dots grown on GaAs with low $T$ photoluminescence (PL) of "as grown" QD's in the range $980-1035 \mathrm{~nm}$. The first type of samples (A) comprises 16 QD layers grown at $T=500{ }^{\circ} \mathrm{C}$ using molecular beam epitaxy (MBE). The dot density is $\approx 6 \times 10^{10} \mathrm{~cm}^{-2}$. In the samples of type A with nominally InAs dots (considered in detail below) each dot layer (2.2 ML thick) is grown in the middle of a $20 \mathrm{~nm}$ GaAs quantum well clad by $10 \mathrm{~nm}$ thick AlGaAs barriers. Different sections of the wafer were thermally annealed at $700{ }^{\circ} \mathrm{C}$ for up to $t_{\mathrm{an}}=17.5 \mathrm{~min}$. In addition, we studied 7 ML thick $\operatorname{In}_{0.5} \mathrm{Ga}_{0.5}$ As dots (low $T$ emission at $1035 \mathrm{~nm}$ ) embedded in Al-free GaAs barriers (type B), and samples of type C, DWELL laser structures grown by MBE. The DWELL structures contain three layers of nominally InAs dots grown between two $\operatorname{In}_{0.15} \mathrm{Ga}_{0.85} \mathrm{As}$ strain-reducing layers ( $2 \mathrm{~nm}$ below and $6 \mathrm{~nm}$ above the dots) with the low $T$ PL peak at $\approx 1200 \mathrm{~nm}$. The dot density is $3.6 \times 10^{10} \mathrm{~cm}^{-2}$.

Differential transmission signals were measured using lock-in techniques. ${ }^{10}$ The radiation was supplied by a femtosecond Ti-sapphire laser and a synchronously pumped optical parametric oscillator (OPO) with repetition rate $76 \mathrm{MHz}$ and emission FWHM of $\approx 10 \mathrm{meV}$ (approximately $200 \mathrm{fs}$ pulse duration). The excitation pulses were tuned into reso-

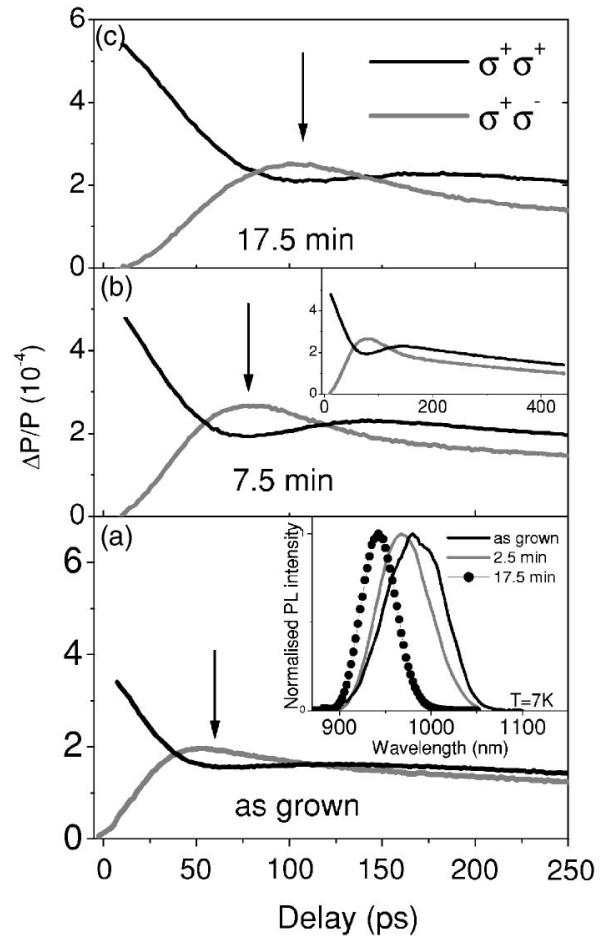

FIG. 2. Dependence of the differential transmission $\Delta P / P$ on the pump-probe delay measured at $T=7 \mathrm{~K}$ for $\sigma^{+}$polarized pump and $\sigma^{+}$or $\sigma^{-}$polarized probe (black and gray lines, respectively). Both fs pulsed are tuned to the QD ground state. The curves are measured for as grown sample (a) and samples after 7.5 and $17.5 \mathrm{~min}$ annealing at $700{ }^{\circ} \mathrm{C}$ [Fig. 2(b) and Fig. 2(c), respectively]. The vertical arrows mark the shift in oscillation time. The inset in Fig. 2(b) shows the traces from Fig. 2(b) on a larger timescale. The inset in Fig. 2(a) shows normalized PL spectra ( $T=7 \mathrm{~K})$ for the unannealed sample with nominally InAs dots (black curve) and samples annealed at $700{ }^{\circ} \mathrm{C}$ for 2.5 and 17.5 (grey curve and symbols, respectively).

nance with the peak of the QD exciton ground state. The results reported are obtained in the low density limit of $<0.1$ excitons per dot.

The inset in Fig. 2(a) shows the results of the PL characterization (at $T=7 \mathrm{~K}$ ) of annealed samples of type A. The PL spectrum measured for the "as grown" sample exhibits a peak at $980 \mathrm{~nm}$ with a FWHM of $77 \mathrm{~nm}(\approx 100 \mathrm{meV})$. After annealing for $2.5 \mathrm{~min}$ at $700{ }^{\circ} \mathrm{C}$ the PL peak shifts to $967 \mathrm{~nm}$ and the width reduces by $10 \%$. As the annealing time is further increased, the PL peak shifts to shorter wavelength and reaches $942 \mathrm{~nm}$ (FWHM $63 \mathrm{meV}$ ) for the sample annealed for $17.5 \mathrm{~min}$. The observed behavior arises due to In/Ga intermixing, ${ }^{24,25}$ which leads to a more Ga rich dot composition and hence weaker confinement potential.

Figure 2 shows $\Delta P / P$ pump-probe traces measured at $T$ $=7 \mathrm{~K}$ for the annealed samples with co- and cross-circularly polarized pulses. The dynamics measured for the as grown sample and samples annealed for $t_{\text {an }}=7.5$ and $17.5 \mathrm{~min}$ are shown in Figs. 2(a)-2(c) respectively. In the $\sigma^{+} \sigma^{+}$configuration the $\Delta P / P$ signal is maximum at zero delay between the pump and probe pulses. The signal exhibits a fast oscillation and then decays with a time constant of few hundred ps at delay times $\tau_{d}>200$ ps [see the inset of Fig. 2(b) for 


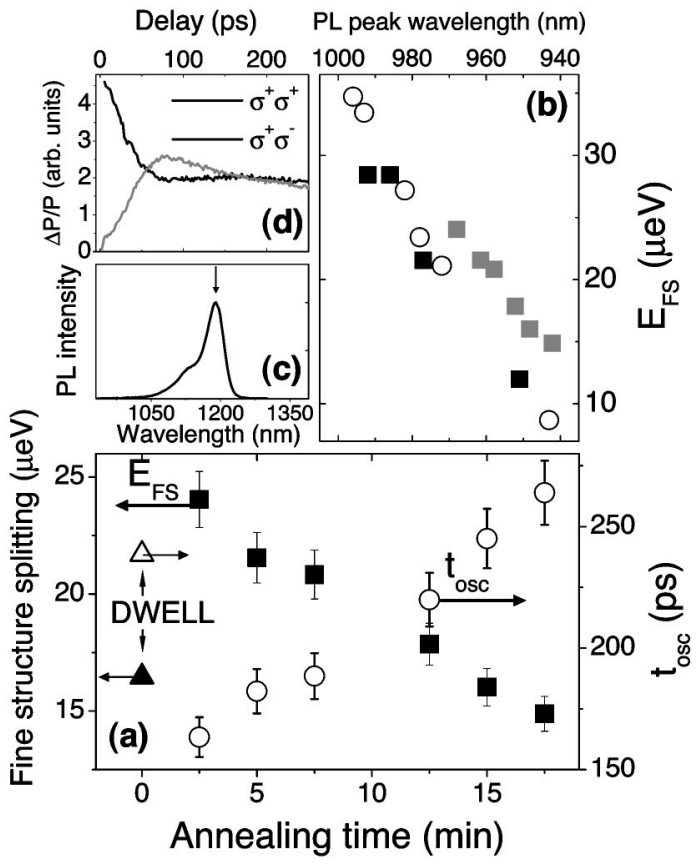

FIG. 3. (a) Dependences on annealing time of the oscillation period $t_{\text {osc }}$ [circles, obtained from fitting of data in Fig. 2 by Eq. (1)] and fine structure splitting $E_{\mathrm{FS}}$ (squares), obtained for the sample with nominally InAs dots grown on GaAs. Triangles show the results for a DWELL structure. (b) $E_{\mathrm{FS}}$ versus PL peak wavelength for $3 \mathrm{In}(\mathrm{Ga})$ As dot samples annealed at 700,750 , and $800{ }^{\circ} \mathrm{C}$ (see text for details). (c) PL spectrum of a DWELL structure. (d) Dependence of the differential transmission $\Delta P / P$ as in Fig. 2 measured at $T=7 \mathrm{~K}$ for the DWELL structure from Fig. 3(c). Both fs pulses are tuned to the QD ground state indicated by the arrow in Fig. 3(c).

pump-probe curves on a longer time scale]. The behavior in the case of the $\sigma^{+} \sigma^{-}$configuration is in antiphase to that of the $\sigma^{+} \sigma^{+}$traces at $\tau_{d}<200 \mathrm{ps}$, with a negligible signal at $\tau_{d} \approx 0 .{ }^{9,10}$

As seen from Fig. 2 a systematic variation of the exciton dynamics is observed in the pump-probe curves measured from samples annealed for different times: As indicated by the vertical arrows, the oscillations in both $\sigma^{+} \sigma^{+}$and $\sigma^{+} \sigma^{-}$ configurations occur on a progressively longer time scale as the annealing time is increased. The increase of the oscillation period corresponds to a reduction in $E_{\mathrm{FS}}$. The oscillation period $t_{\mathrm{osc}}$ (and hence $E_{\mathrm{FS}}=h / t_{\mathrm{osc}}$ ) is deduced from fitting of the $\Delta P / P$ traces using Eq. (1). The dependences of $t_{\mathrm{osc}}$ and $E_{\mathrm{FS}}$ on $t_{\mathrm{an}}$ are plotted in Fig. 3(a). After annealing for $17.5 \mathrm{~min} t_{\mathrm{osc}}$ reaches $\approx 260 \mathrm{ps}$, corresponding to $E_{\mathrm{FS}}$ $\approx 15 \mu \mathrm{eV}$.

We find that for the unannealed sample a satisfactory fit of the $\Delta P / P$ curves is only possible if the contributions of at least two dot distributions to the oscillation behavior are taken into account. This is consistent with the observation of multi-modal structure in the PL spectrum of the dot ensemble in the as grown sample (Fig. 2). The annealing results in suppression of the multi-modal dot distribution in the PL spectra, which we are able to confirm also on a very fine energy scale in the pump-probe measurements.

Figure 3(b) compares the results presented in Fig. 3(a) with $E_{\mathrm{FS}}$ data measured on two other samples of type B. $E_{\mathrm{FS}}$ is plotted in Fig. 3(b) versus the PL peak wavelength $\lambda_{\mathrm{PL}}$ which decreases with annealing time for these samples. The data from Fig. 3(a) (for the nominally InAs QD sample of type A) is shown by grey squares, while the results for two samples of type B are shown by open and black symbols, respectively. These samples were annealed for up to $20 \mathrm{~min}$ at $750^{\circ}$ (points above $970 \mathrm{~nm}$ ) and $800^{\circ} \mathrm{C}$ (points below $950 \mathrm{~nm}$ ). As seen, $E_{\mathrm{FS}}$ can be tuned by annealing over a very large range from $\approx 36$ to $8 \mu \mathrm{eV}$. The almost linear correlation between $\lambda_{\mathrm{PL}}$ and $E_{\mathrm{FS}}$ for all three samples is notable, however, a difference is observed in the dependences $E_{\mathrm{FS}}\left(\lambda_{\mathrm{PL}}\right)$ for different types of samples. The sample with nominally InAs dots has larger $E_{\mathrm{FS}}$ in the range of $\lambda_{\mathrm{PL}}$, where the dependences from the different samples overlap: for $\lambda_{\mathrm{PL}} \approx 950 \mathrm{~nm} E_{\mathrm{FS}}$ measured for this sample is up to a factor of 2 higher than for the samples with nominally $\mathrm{In}_{0.5} \mathrm{Ga}_{0.5} \mathrm{As}$ dots.

One of the important parameters which has an impact on $E_{\mathrm{FS}}$ and is modified by the In/Ga intermixing during annealing is strain. During the growth of quantum dots the strain due to the lattice mismatch with the underlying GaAs is relieved partially via the coarsening of the In-rich surface and eventual formation of dots. However, the consequent capping of In-rich dots with GaAs results in additional compressive strain in dots and tensile strain in GaAs. ${ }^{16,17}$ These strains will be reduced during thermal annealing, ${ }^{25}$ producing more Ga-rich dots due to the In/Ga intermixing and thus reducing the lattice constant contrast in the material surrounding the dots. The reduction of strain (with the latter predicted to induce further anisotropy in dots ${ }^{16}$ ) will result in more $s$-like exciton wave functions and hence weaker $e-h$ exchange interaction. ${ }^{15}$

In order to further investigate the validity of our suggestions we have studied a dot-in-a-well structure (sample C), where the dots are surrounded by material with considerably smaller lattice contrast: InAs dots are grown inside an $\mathrm{In}_{0.15} \mathrm{Ga}_{0.85} \mathrm{As}$ layer. Strain engineering by capping of dots with InGaAs layers is well documented. ${ }^{21}$ It has been found that covering dots with $4 \mathrm{~nm}$ of $\operatorname{In}_{x} \mathrm{Ga}_{1-x} \mathrm{As}$ results in a marked red-shift of the dot emission, with the strain reduction in the dots playing a major role in the lowering of the transition energy (to a wavelength of $\approx 1.3 \mu \mathrm{m}$ at $T$ $=300 \mathrm{~K}$ in the dots we study).

Figure 3(c) shows a PL spectrum for the DWELL structure investigated, with the arrow indicating the ground state spectral position $(\approx 1190 \mathrm{~nm})$ where the pump-probe signal is measured. In the same way as the data in Fig. 2, the differential transmission signal in both polarization configurations shows oscillations [Fig. 3(d)], evolving relatively slowly as compared with "as grown" conventional dot samples [Fig. 2(a)]. Analysis of the results using Eq. (1) provides the data shown in Fig. 3(a) by triangles: $t_{\mathrm{osc}}$ $=238 \mathrm{ps}$ and $E_{\mathrm{FS}}=16.5 \mu \mathrm{eV}$. To our knowledge, the data for $E_{\mathrm{FS}}$ reported here is the first measurement of the fine structure splitting for DWELL structures.

The magnitude of $E_{\mathrm{FS}}$ is about 2 times smaller than that detected for unannealed dots grown on GaAs. This deviates notably from the trend in $E_{\mathrm{FS}}\left(\lambda_{\mathrm{PL}}\right)$ observed in Fig. 3(b), showing that the magnitude of the exciton fine structure splitting is not directly related to the magnitude of confine- 
ment of the electron and hole. On the other hand, the small magnitude of $E_{\mathrm{FS}}$ in the strain-engineered dot structure supports the effect of strain reduction on decreasing the size of the electron-hole exchange interaction. ${ }^{27}$

In summary, experimental evidence is found for the effect of strain reduction on the magnitude of the fine structure splitting of quantum dot excitons. The splitting is shown to decrease in dots after annealing and also in strain-engineered dot-in-a-well structures. During annealing In/Ga intermixing leads to smaller lattice constant contrast in the dot environment, leading to strain reduction. Similarly, in dot-in-a-well structures strain is reduced since dots are surrounded by InGaAs layers with a more similar lattice constant to the In-rich dots. Comparative studies of several $\mathrm{In}(\mathrm{Ga}) \mathrm{As}$ dot structures also indicate that exciton confinement has no direct effect on the fine structure splitting. Finally, thermal annealing is shown to be a suitable tool to produce small fine structure splittings required for sources of entangled photons based on individual dots.

During the final stage of the preparation of this paper we became aware that similar effects of annealing measured using four-wave-mixing techniques have been reported by another group. ${ }^{28}$ However, no detailed discussion as to the origin of this observation has been presented.

This work has been supported by EPSRC Grant No. GR/ S49308 and by the Nanomat Project of the Growth Programme of the European Community Grant No. G5RD-CT2001-00545 and by the Royal Society.
${ }^{1}$ D. Gammon, E. S. Snow, B. V. Shanabrook, D. S. Katzer, and D. Park, Phys. Rev. Lett. 76, 3005 (1996).

${ }^{2}$ N. H. Bonadeo, J. Erland, D. Gammon, D. Park, D. S. Katzer, and D. G. Steel, Science 282, 1473 (1998).

${ }^{3}$ T. Flissikowski, A. Hundt, M. Lowisch, M. Rabe, and F. Henneberger, Phys. Rev. Lett. 86, 3172 (2001).

${ }^{4}$ M. Bayer, A. Kuther, A. Forchel, A. Gorbunov, V. B. Timofeev, F. Schafer, J. P. Reithmaier, T. L. Reinecke, and S. N. Walck, Phys. Rev. Lett. 82, 1748 (1999).

${ }^{5}$ V. D. Kulakovskii, G. Bacher, R. Weigand, T. Kümmell, A. Forchel, E. Borovitskaya, K. Leonardi, and D. Hommel, Phys. Rev. Lett. 82, 1780 (1999).

${ }^{6}$ M. Bayer, G. Ortner, O. Stern, A. Kuther, A. A. Gorbunov, A. Forchel, P. Hawrylak, S. Fafard, K. Hinzer, T. L. Reinecke, S. N. Walck, J. P. Reithmaier, F. Klopf, and F. Schäfer, Phys. Rev. B 65, 195315 (2002), and references therein.

${ }^{7}$ J. J. Finley, D. J. Mowbray, M. S. Skolnick, A. D. Ashmore, C. Baker, A. F. G. Monte, and M. Hopkinson, Phys. Rev. B 66, 153316 (2002).

${ }^{8}$ G. Bester, S. Nair, and A. Zunger, Phys. Rev. B 67, 161306(R) (2003).

${ }^{9}$ A. S. Lenihan, M. V. Gurudev Dutt, D. G. Steel, S. Ghosh, and P. K. Bhattacharya, Phys. Rev. Lett. 88, 223601 (2002).

${ }^{10}$ A. I. Tartakovskii, J. Cahill, M. N. Makhonin, D. M. Whittaker, J-P. R. Wells, A. M. Fox, D. J. Mowbray, M. S. Skolnick, K. M. Groom, M. J. Steer, and M. Hopkinson, Phys. Rev. Lett. 93, 057401 (2004).

${ }^{11}$ C. Santori, D. Fattal, M. Pelton, G. S. Solomon, and Y. Yamamoto, Phys. Rev. B 66, 045308 (2002).

${ }^{12}$ O. Benson, C. Santori, M. Pelton, and Y. Yamamoto, Phys. Rev. Lett. 84, 2513 (2000).

${ }^{13}$ P. Borri, W. Langbein, S. Schneider, U. Woggon, R. L. Sellin, D. Ouyang, and D. Bimberg, Phys. Rev. Lett. 87, 157401 (2001); D. Birkedal, K. Leosson, and J. M. Hvam, ibid. 87, 227401 (2001).

${ }^{14}$ C. Bennett and D. DiVincenzo, Nature (London) 404, 247
(2000).

${ }^{15}$ T. Takagahara, Phys. Rev. B 47, 4569 (1993); 62, 16840 (2000).

${ }^{16}$ John H. Davies, J. Appl. Phys. 84, 1358 (1998); A. D. Andreev, J. R. Downes, D. A. Faux, and E. P. O'Reilly, ibid. 86, 297 (1999).

${ }^{17}$ M. Grundmann, O. Stier, and D. Bimberg, Phys. Rev. B 52, 11969 (1995); O. Stier, M. Grundmann, and D. Bimberg, ibid. 59, 5688 (1999).

${ }^{18}$ A. Franceschetti, H. Fu, L. W. Wang, and A. Zunger, Phys. Rev. B 60, 1819 (1999).

${ }^{19}$ G. Dasbach, A. A. Dremin, M. Bayer, V. D. Kulakovskii, N. A. Gippius, and A. Forchel, Phys. Rev. B 65, 245316 (2002); Physica E (Amsterdam) 13, 394 (2002).

${ }^{20}$ M. O. Lipinski, H. Schuler, O. G. Schmidt, K. Eberl, and N. Y. Jin-Phillipp, Appl. Phys. Lett. 77, 1789 (2000); see also, O. G. Schmidt and K. Eberl, Phys. Rev. B 61, 13721 (2000), for results on $\mathrm{Ge} / \mathrm{Si}$ dots.

${ }^{21}$ F. Guffarth, R. Heitz, A. Schliwa, O. Stier, N. N. Ledentsov, A. R. Kovsh, V. M. Ustinov, and D. Bimberg, Phys. Rev. B 64, 085305 (2001), and references therein.

${ }^{22}$ H. Y. Liu, M. Hopkinson, C. N. Harrison, M. J. Steer, R. Frith, I. R. Sellers, D. J. Mowbray, and M. S. Skolnick, J. Appl. Phys. 93, 2931 (2003).

${ }^{23}$ Masaharu Mitsunaga and C. L. Tang, Phys. Rev. A 35, 1720 (1987).

${ }^{24}$ S. Fafard and C. Ni. Allen, Appl. Phys. Lett. 75, 2374 (1999).

${ }^{25}$ C. Lobo, R. Leon, S. Fafard, and P. G. Piva, Appl. Phys. Lett. 72, 2850 (1998).

${ }^{26}$ We assume equal distribution of carriers captured in dots between spin up and spin down populations.

${ }^{27}$ The magnitude of the fine structure splitting in QD lasers can also be affected by the high $T\left(>600^{\circ} \mathrm{C}\right)$ growth of the AlGaAs waveguide cladding leading effectively to the slight annealing of the dots.

${ }^{28}$ W. Langbein, P. Borri, U. Woggon, V. Stavarache, D. Reuter, and A. D. Wieck, Phys. Rev. B 69, 161301(R) (2004). 